\title{
Effects of different wall materials on the physicochemical properties and oxidative stability of spray-dried microencapsulated red-fleshed Pitaya (Hylocereus polyrhizus) seed oil.
}

\begin{abstract}
The aim of this research was to investigate the influence of the composition of the wall material on the encapsulation and stability of microencapsulated red-fleshed pitaya seed oil. Hylocereus polyrhizus seed oil was homogenized with various wall material solutions at a core/wall material ratio of 0.33 and was microencapsulated by spray-drying. The microstructure and morphology of pitaya seed oil powder (PSOP) were observed using a scanning electron microscope (SEM). PSOP encapsulated with gum Arabic exhibited a lower degree of microencapsulation efficiency (MEE; 77.61-85.3\%) compared to PSOP encapsulated with proteinaceous bases $(90.12-98.06 \%)$. The study on oil retention revealed that sodium caseinate $>$ whey protein $>$ gum Arabic as effective wall materials for pitaya seed oil encapsulation. The effects of different wall systems on the oxidation stability of PSOP were studied under accelerated storage conditions; the peroxide value (POV) was determined throughout the test interval at several storage times. This study indicates that the use of lactose as wall material is able to increase the oxidation stability of PSOP; however, further research is needed to evaluate its antioxidative retention toward the oxidative stability of PSOP.
\end{abstract}

Keyword: Dragon fruit; Hylocereus polyrhizus; Pitaya; Seed oil; Microencapsulation; Spray-drying; Lipid oxidation. 